PVP2002-1580

\title{
NUMERICAL ANALYSIS ON WAVE DYNAMIC PROCESSES IN PULSE DETONATION DEVICES
}

\author{
Z.L. Jiang, C. Wang, Z.M. Hu and W. Zhao \\ Key Laboratory of High Temperature Gas Dynamics \\ Institute of Mechanics, Chinese Academy of Sciences \\ 15 Zhong Guan Cun Road, Beijing 100080, China \\ zljiang@imech.ac.cn
}

\begin{abstract}
In this paper, wave dynamics processes occurring in pulse detonation devices are analyzed both numerically and experimentally, including the propagation of detonation fronts, the motion of rarefaction waves in gas exhausting phase and the diffraction of shock waves at thrust nozzles. Numerical results are also compared with experiments to confirm the observed wave phenomena. In order to estimate operation roles of pulse detonation engines more accurately, the initiation of the air/hydrogen mixture is also examined experimentally at certain conditions. Numerical analysis indicates that pulse detonation devices can be operated in a quite high frequency, the gas-filling phase occupies a longer time in one detonation cycle, and decreasing the length of detonation chambers for the higher operation frequency is possible, but has its limitation.
\end{abstract}

\section{INTRODUCTION}

The idea of detonation came into being after disastrous explosions in colamine were reported (Nettleton, 1987). People were puzzling how the slow subsonic combustion could produce such the strong mechanic effect. Understanding came after discovering that the detonation of combustible mixtures is close to the constantvolume process, in which much more mechanic energy could be generated than in the constant-pressure combustion occurring in conventional engines, such as turbojets, turbofans, ramjets and air-turbo rockets. Motivated by the pulse-jet propulsion, the research on detonation applications in engines was initiated early up to 1941 (Doring, 1943; Hoffmann, 1940; Nicholls et al, 1957), but eased twenty years later because of the lack of understanding on detonation phenomena (Krzychi, 1962). However, during the recent years, there has been a growing interest in exploring detonation physics for efficient propulsion systems to meet the increasing requirements of high-speed vehicles, for example, the pulse detonation engines (PDEs) and other PDE-based propulsion systems (Heldman et al., 1986; Hinkey et al., 1995; Bussing et al., 1994; Brophy et al., 1998; Kailasanath, 2000). The PDEs have many desirable features, such as high specific thrust, low specific fuel consumption, simple configuration and high thermodynamic efficiency. Additionally, it does not necessarily require a compressor or a turbine, and is capable of operating in both the self-breathing or the rocket mode. Analysis based on numerical simulations has shown that it can be operated in a wide flight environment from subsonic to supersonic (Kailasanath, 2000). Because of these potential advantages, it is expected that the successful development of the PDEs will lead to a revolution in the thermal engine research field. However, there are no practical propulsion systems based on detonation because of the difficulties involved in rapidly mixing of the fuel and air in the filling phase, fast initiating of a self-sustaining detonation in a controlled manner and efficiently protecting the fresh gas from auto-ignition due to high temperature exhausted gases. These difficulties are closely related to the wave dynamic process occurring in pulse detonation cycles, including gas filling and mixing, the DDT process, detonation propagation and rarefaction wave motion. Such the dynamic precesses play important role in determining a PDE cycle. 
In this paper, the above-mentioned wave dynamic processes are simulated numerically by solving the Euler equations for a perfect gas mixture implemented with a pseudo-kinetic reaction model. Some obtained results are verified with experiments. The wave processes studied here include the propagation of detonation fronts, the motion of rarefaction waves in gas exhausting phase and the diffraction of the leading shock wave at thrust nozzles. In order to estimate the operation roles of pulse detonation engines more accurately, the DDT process related to initiation of the air/hydrogen mixture is also examined experimentally at certain conditions.

\section{NUMERICAL METHOD}

The physic phenomena concerned in this paper are the wave dynamic processes in which viscosity effects are negligible. The two-dimensional Euler equations in conservation form for a binary mixture of the perfect gases are accepted in the present numerical simulations and can be written in cylindrical coordinates as

$$
\frac{\partial \mathbf{U}}{\partial t}+\frac{\partial \mathbf{F}}{\partial x}+\frac{\partial \mathbf{G}}{\partial r}+\frac{1}{r} \mathbf{S}=\mathbf{H},
$$

where $(x, r, t)$ are coordinates and time, and $\mathbf{U}, \mathbf{F}, \mathbf{G}$, $\mathbf{S}$ and $\mathbf{H}$ denote the state variable, flux and sources, respectively, given by

$$
\begin{gathered}
\mathbf{U}=\left(\begin{array}{c}
\rho_{1} \\
\rho_{2} \\
\rho u \\
\rho v \\
e
\end{array}\right), \quad \mathbf{F}=\left(\begin{array}{c}
\rho_{1} u \\
\rho_{2} u \\
\rho u^{2}+p \\
\rho u v \\
(e+p) u
\end{array}\right), \quad \mathbf{G}=\left(\begin{array}{c}
\rho_{1} v \\
\rho_{2} u \\
\rho u v \\
\rho v^{2}+p \\
(e+p) v
\end{array}\right), \\
\mathbf{S}=\left(\begin{array}{c}
\rho_{1} v \\
\rho_{2} u \\
\rho u v \\
\rho v^{2} \\
(e+p) v
\end{array}\right), \quad \mathbf{H}=\left(\begin{array}{c}
\rho h\left(\rho_{1}, \rho_{2}, p\right) \\
-\rho h\left(\rho_{1}, \rho_{2}, p\right) \\
0 \\
0 \\
0
\end{array}\right), \quad(2)
\end{gathered}
$$

where primitive variables in the unknown $U$ are density $\rho$; the reactant density $\rho_{1}$; the product density $\rho_{2}$; velocity components $u$ and $v$ in $x$ - or $y$-direction, respectively; the reaction rate $h\left(\rho_{1}, \rho_{2}, p\right)$; and total energy per unit mass $e$, related to the equation of state for the perfect gaseous mixture by

$$
e=\frac{p}{\gamma-1}+\frac{1}{2} \rho\left(u^{2}+v^{2}\right)+\rho_{1} Q,
$$

where $Q$ is the chemical reaction heat per unit mass. It is assumed that the equation of state is independent of the composition of gas mixtures, but the internal specific energy is modified to include the latent heat of combustion. The density of the gas mixture is calculated by

$$
\rho=\rho_{1}+\rho_{2} \text {. }
$$

If mass fractions are denoted by $Z_{1}=\rho_{1} / \rho$ and $Z_{2}=$ $\rho_{2} / \rho$, the effective adiabatic exponent of the gas mixture, $\gamma$, can be put in the form

$$
\gamma=\frac{\frac{Z_{1} \gamma_{1}}{m_{1}\left(\gamma_{1}-1\right)}+\frac{Z_{2} \gamma_{2}}{m_{2}\left(\gamma_{2}-1\right)}}{\frac{Z_{1}}{m_{1}\left(\gamma_{1}-1\right)}+\frac{Z_{2}}{m_{2}\left(\gamma_{2}-1\right)}}
$$

where $\gamma_{1}$ and $m_{1}$ are the adiabatic exponent and molecular weight for the reactant, and $\gamma_{2}$ and $m_{2}$ are the respective parameters for the product. The governing equations, Eqs. (1), are discretized using a dispersioncontrolled scheme proposed by Jiang et al. (1995). The time-marching integration is performed using a Runge-Kutta integration method of second-order accuracy. The computational mesh uses an equally-spaced Cartesian grid to accommodate the oblique rigid wall of thrust nozzles, where the ratio of mesh size $\Delta y / \Delta x$ is chosen so that the oblique wall could coincide with the cell diagonal. This choice enables a simple and accurate algorithm to be implemented with our scheme, producing a rigid wall boundary condition of secondorder accuracy through computing the "mirror-image" flow states at virtual grid points outside the wall.

\section{REACTION MODEL}

In order to examine wave processes in pulse detonation engines, the entire pulse detonation engine has to be simulated. According to the computer sources available the mesh size that could be used is still too large to capture accurately either the detonation structure or the thickness of detonation front because of the large lengthto-diameter ratio of the PDEs configurations. Therefore, the strategy for selecting a kinetic reaction rate model is to obtain the Taylor expansion wave having an infinitely thin reaction zone, which are known to predict well the global features of gaseous detonation. The emphasize here is to predict the gas dynamics in front of or following a detonation front not to correctly produce either the structure of the detonation front or the process by which a shock wave propagating in the reactive gas mixture evolves gradually into a detonation wave. The following pseudo-kinetic reaction rate (Jiang et al., 1997) has been demonstrated to be acceptable for our 
purposes.

$$
h\left(\rho_{1}, \rho_{2}, p\right)= \begin{cases}0 & p<P_{i g n i t} \\ -A \operatorname{det}\left(\frac{D_{C J}}{\Delta s}\right)\left(\frac{p}{P_{C J}}\right)^{\alpha} Z_{1}^{\beta} & \text { otherwise }\end{cases}
$$

where $D_{C J}$ is the C-J velocity; $P_{C J}$ is the C-J pressure; $\Delta s$ is the local mesh size; $P_{i g n i t}$ is an ignition pressure; Adet, $\alpha, \beta$ are dimensionless constants of order unity whose values are determined by inspecting onedimensional simulations of detonation and looking for the captured solutions close to the Taylor similar solutions. A set of values for these constants was determined by such a procedure and is given below by Jiang et al. (1997).

$$
\left\{\begin{array}{l}
P_{\text {ignit }}=0.4 P_{C J}, \\
\alpha=1.0 \\
\beta=0.5 \\
\text { Adet }=1.6 .
\end{array}\right.
$$

All the computational results presented here have been produced with this set of the "tuned" constants of the pseudo-kinetic reaction model. Validation of this model has been carried out by comparing numerical results with experimental data, and the maximum discrepancy in the C-J velocity, pressure and Mach number is less than 5\%, which is in an acceptable level for analyzing the wave dynamic processes.

\section{EXPERIMENTAL FACILITY}

Experiments were carried out by using a single-tube PDE experimental facility as schematically shown in Fig. 1, which was manufactured and installed in the Key Laboratory of High Temperature Gas Dynamics, Institute of Mechanics, Chinese Academy of Sciences, Beijing. This facility mainly consists of a detonation chamber, a DDT-enhanced combustion chamber, a dump tank, and a high-power electric igniter. The detonation chamber has a bore of $68 \mathrm{~mm}$ and a length of $4 \mathrm{~m}$. Three pressure sensors are placed at position $A, B, C$ as shown in Fig. 1. The distance is $350 \mathrm{~mm}$ between the igniter and position $A, 1550 \mathrm{~mm}$ between position $A$ and $B$ and $2.0 \mathrm{~m}$ between position $B$ and $C$. There are five ionization probes distributed equally between position $A$ and $B$ to measure detonation wave speeds.

In experiments, hydrogen is used as the fuel, and air or oxygen is the oxidizer. All the mixtures are in stoichiometric proportion. Two kinds of the igniters used are the electric wire with the ignition energy being about 10 $J$ per pulse and the high-energy spark plug with the ignition energy of $2 J$ per pulse. Some special devices were

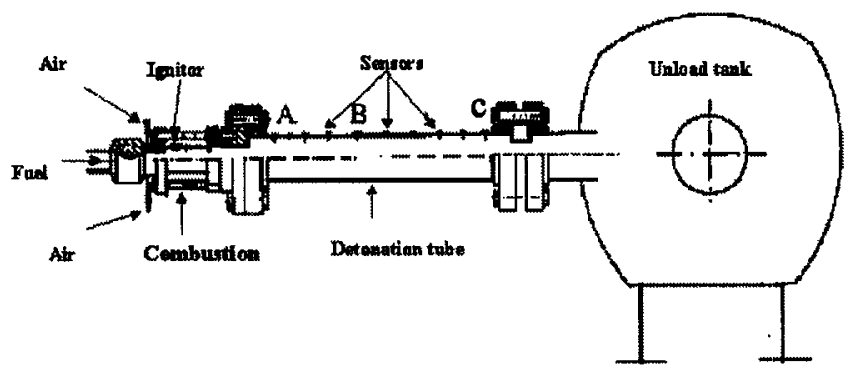

Figure 1: Schematic of the PDE experiment facility for study of wave dynamic processes in Institute of Mechanics, Beijing

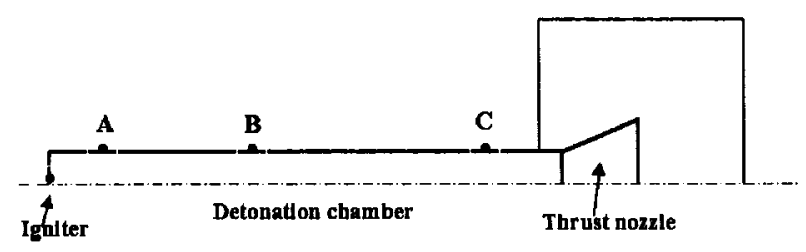

Figure 2: Simplified computational domain for PDE simulations

applied in the combustion chamber to enhance fuel/air mixing and wave interactions.

\section{NUMERICAL RESULTS AND DISCUSSION}

Considering the PDE experiment facility as introduced in the last section, a simplified computational domain is shown in Fig. 2, where it consists of three parts: the detonation chamber, the thrust nozzle and an additional part containing the thrust nozzle for overcoming the difficulty in boundary condition specification. The detonation chamber is $4 \mathrm{~m}$ in length and $68 \mathrm{~mm}$ in diameter. The thrust nozzle angel can vary from $15^{\circ}$ to $60^{\circ}$ degree. The diameter of the additional part is taken to be 6 times larger than the diameter of the detonation chamber. The multi-block technique is used to deal with the complex grid system.

In numerical simulations, the reflecting boundary is specified on rigid walls and along the axis of symmetry. The non-reflecting condition is used on the outflow boundary. For detonation ignition, it is realized by prescribing a small high temperature and pressure region at the closed end of the detonation chamber. Actually, the DDT process is too difficult to be numerically simulated because of something uncertain in experiments, therefore, will be examined experimentally in this paper to investigate its effects on the PDEs cycle. 

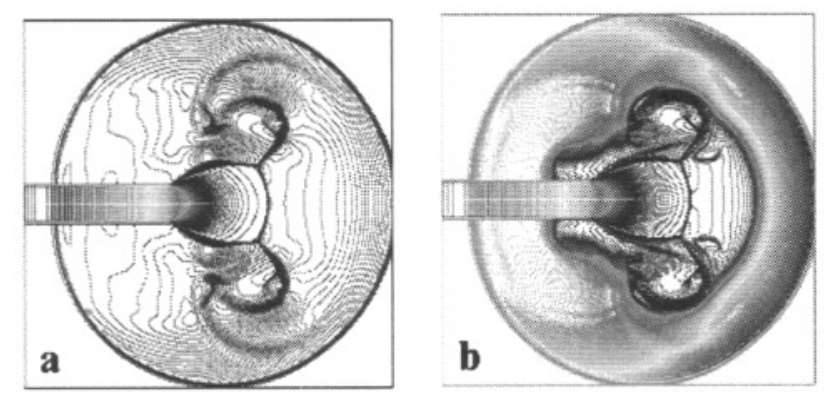

Figure 3: Shock wave diffraction at the open end of the detonation chamber: $\mathbf{a}$ pressure isobars; $\mathbf{b}$ density distribution, at the initial conditions of air: $\mathrm{H}_{2}=2.46$ and $P_{4 i}=0.2 M P a$.

\section{Wave Processes in the PDE Facility}

One PDE cycle consists of three main dynamic processes: they are the detonation propagation, the rarefaction wave motion in the exhausting process and the filling of detonative gas mixtures. The filling process is an aerodynamic problem, therefore, only the first two processes are considered to be wave dynamic ones and will be investigated in the present study.

Figure 3 shows the leading shock wave discharging out of the detonation chamber without thrust nozzles. The rate of air to hydrogen is taken as 2.46 and the gas mixture is pressurized at an initial pressure of $0.2 M P a$. The detonation process is completed at the open end of the detonation tube out of which shock wave diffraction develops. Isobars of the shock wave diffraction are plotted in Fig. 3a and the density distribution is given in Fig. 3b. From theses figures it is observed that rarefaction waves were moving into the detonation chamber while the shock wave discharged out of the detonation chamber. The rarefaction waves lead the gas exhausting process, and the shock wave diffraction results in a pressure drop and an exhausted gas acceleration.

The pressure history numerically simulated at position $B$ being $1.9 \mathrm{~m}$ downstream from the closed end of the detonation chamber is plotted in Fig. 4 with an experimental result (colored line) together. The experimental result is obtained at the similar condition as the numerical one. The maximum discrepancy in the $\mathrm{C}$ $\mathrm{J}$ velocity, pressure and Mach number is less than $5 \%$, which is in an acceptable level for analyzing the wave dynamic processes. From this figure, a peak pressure is observed, which indicates the arrival of the detonation front. The peak pressure decreases very quickly due

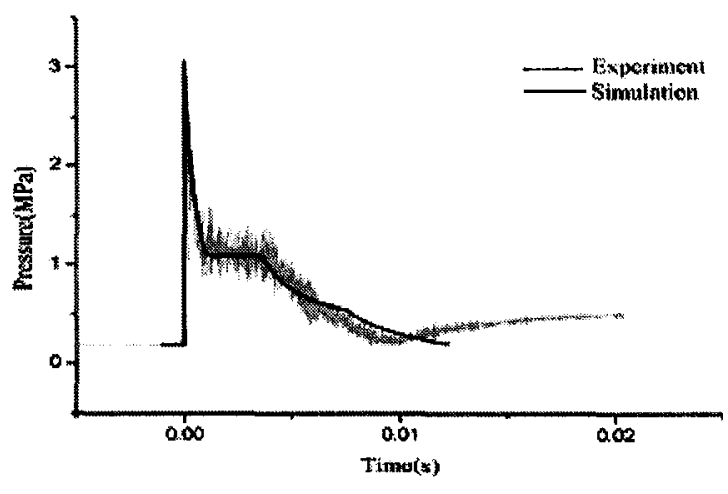

Figure 4: Pressure histories measured at position $B$ being $1.9 \mathrm{~m}$ downstream from the igniter: numerical result (black line) and experimental data (colored line) at the initial conditions of air: $\mathrm{H}_{2}=2.46$ and $P_{4 i}=0.2 \mathrm{MPa}$.

to the Taylor expansion wave and then the flow state reaches to a steady state (a pressure planum). Finally, rarefaction waves propagate over this measurement station and the pressure even decreases more.

Figure 5 shows pressure variations of numerical results at positions $A, B$ and $C$, respectively. From these three curves one can see a pressure jump that indicates the arrival of detonation front, a pressure decrease due to the Taylor expansion wave, a pressure planum the value of which can be determined with the Taylor similarity law at the closed end of the detonation tube, and the second pressure drop because of the arrival of rarefaction waves. In curve $A$, the period of the pressure drop due to the Taylor expansion wave is very short since the position is close to the igniter. There is no uniform pressure in curve $C$, because the rarefaction waves come before the Taylor expansion completes. For this case, the detonation speed is $1935.4 \mathrm{~m} / \mathrm{s}$ for experiment and $1916.4 \mathrm{~m} / \mathrm{s}$ for CFD, and the speed of rarefaction waves is about $872 \mathrm{~m} / \mathrm{s}$ for experiment and $851 \mathrm{~m} / \mathrm{s}$ for CFD. Using these results for a given detonation chamber, the time for both detonation and exhausting processes can be estimated for one PDE cycle. For example, if a detonation chamber is $4 \mathrm{~m}$ long, it takes about less than $6 \mathrm{~ms}$ for these two processes to complete. This rneans that PDEs can be operated at quite higher frequencies that mainly depends on the filling precess.

\section{Wave Diffraction in Thrust Nozzles}

The leading shock wave diffracts downstream along nozzle walls after it discharges from the detonation chamber. The wall pressure will jump to a post-shock 


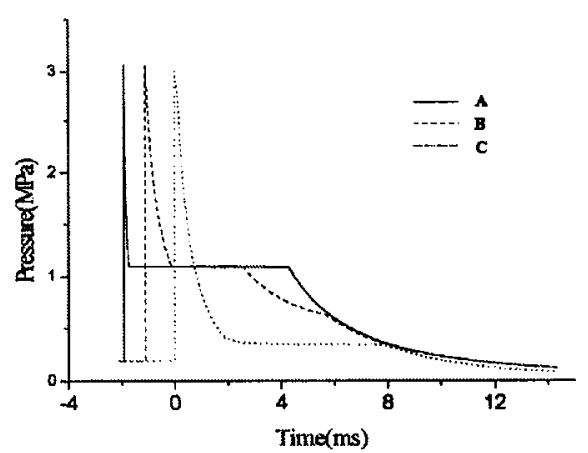

Figure 5: Pressure histories recorded numerically at positions $A, B$ and $C$, respectively, at the initial conditions of air: $\mathrm{H}_{2}=2.46$ and $P_{4 i}=0.2 M P a$.

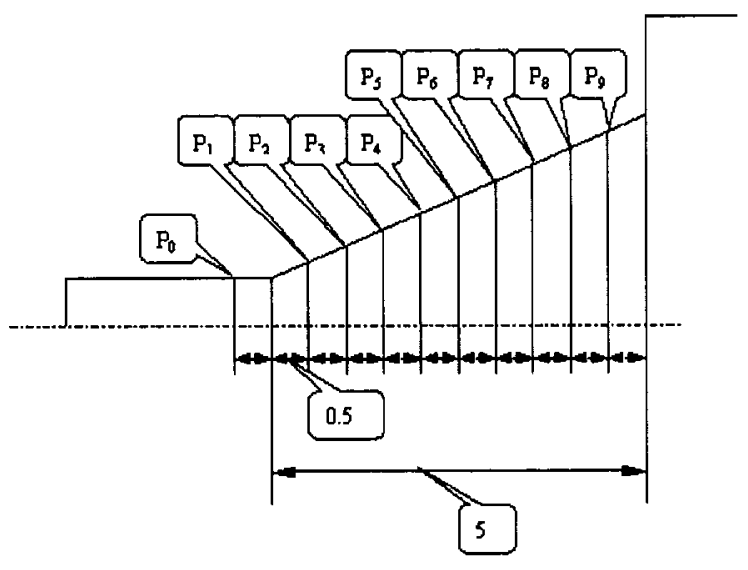

Figure 6: Schematic diagram of nozzle configuration and measurement positions for numerical simulations

pressure but decrease due to nozzle flow expansion. This is the period during which the thrust is generated. In order to understand wave dynamics in nozzle flows and improve the thrust performance of PDEs. Several thrust nozzles with various diverging angles have been simulated numerically and the results of two cases are presented in this paper.

Figure 6 shows a schematic diagram of the thrust nozzle configuration for computation and measurement positions. Ten positions are distributed equally with a distance being half of the detonation chamber diameter. The first position is located inside the detonation chamber to detect the shock pressure.

Figure 7 shows pressure distribution of the flow field when the leading shock wave is diffracting on the noz-

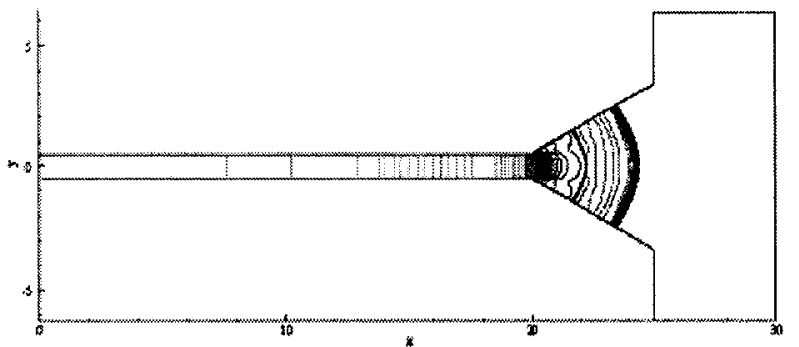

Figure 7: Pressure distribution of the flow field when the leading shock wave diffracts over the thrust nozzle with a $30^{\circ}$ diverging angle

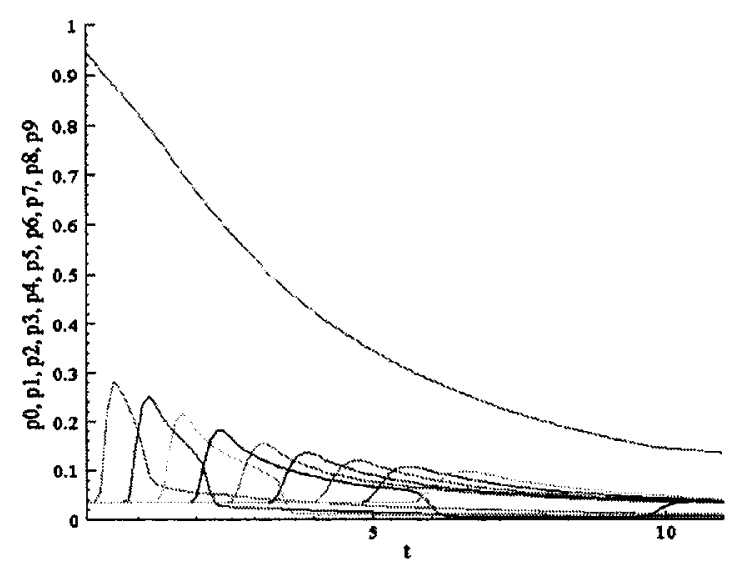

Figure 8: Pressure variations on the nozzle wall when the leading shock wave diffracts over the thrust nozzle with a $30^{\circ}$ diverging angle

zle wall with a $30^{\circ}$ diverging angle. Behind the leading shock wave another shock wave appears, which indicates that the local supersonic flow develops due to nozzle flow expansion. This flow expansion results in a pressure drop to a very low level. Rarefaction waves are also observed and they propagate upstream into the detonation chamber from the detonation chamber exit.

The pressure histories at ten measurement stations are presented in Fig. 8. From this figure, one could find that pressure jumps appear when the leading shock wave arrives at these measurement stations, and then They are getting lower and lower from position 0 to 9 because of shock wave diffraction. The first pressure jump is normalized as a unit and the following pressure jumps are quite low, actually, less than $30 \%$ of the first one if compared with the first. Moreover, these pressure rises last 


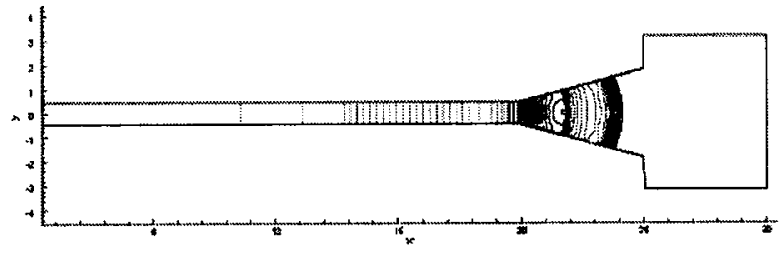

Figure 9: Pressure distribution of the flow field when the leading shock wave diffracts over the thrust nozzle with with a $15^{\circ}$ diverging angle

only for a short time and then decrease to very low level due to the nozzle flow expansion. This indicates that the thrust nozzle does improve the PDE performance but its effects must be evaluated more carefully.

Numerical results calculated for the case with a thrust nozzle of a $15^{\circ}$ diverging angle are presented in Figs. 9 and 10. Figure 9 shows pressure distribution of the nozzle flow when the leading shock wave is diffracting over the thrust nozzle. Figure 10 gives pressure variations at various measurement stations on the thrust wall. Flow phenomena observable in this case are similar to the last case except the higher pressure jumps that are due to the smaller diverging angle. The PDE thrust depends on pressure integration over the whole nozzle wall as well as the lasting time of the pressure jump behind the diffracting shock wave. The thrust performance of PDEs would be evaluated over one PDE cycle, however, the nozzle with a $15^{\circ}$ diverging angle shows a more promising character, that is the higher pressure jump and longer lasting time.

\section{Detonation initiation and the DDT process}

Effects of the DDT precess on PDE cycles are very difficult to account for because it is not only difficult to measure but also may vary case by case because of some experimental uncertain. Moreover, the initial pressure, the gas mixtures and the ignition energy play an important role in it. Figure 11 shows pressure variations measured at position A for two tests at the same initial conditions but with different ignition energy. From this result, it was observed that the DDT process in Fig. 11a completed earlier than in Fig. 11b. This is because the ignition energy used in the case of Fig. 11a is higher. However, the time interval in which the detonation front propagates from position $A$ to $B$ appears almost the same. Considering the detonation speed is much faster than rarefaction waves, and even ten times faster than the gas filling speed, the time difference in-

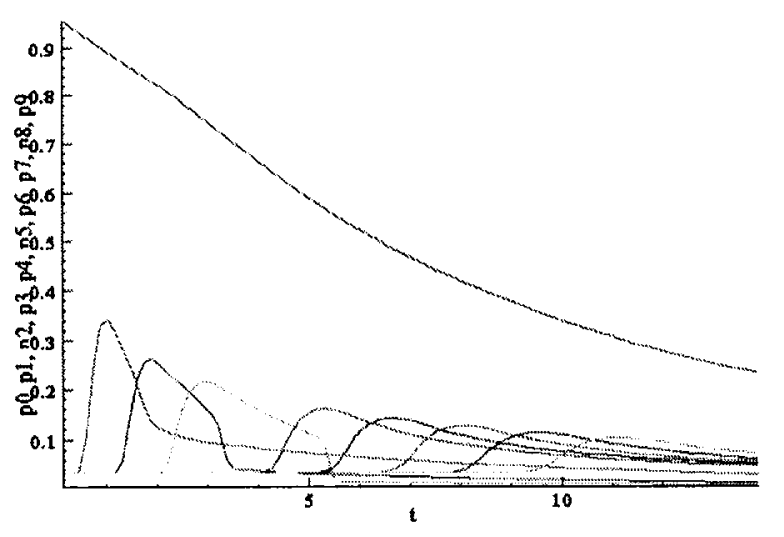

Figure 10: Pressure variations on the nozzle wall when the leading shock wave diffracts over the thrust nozzle with a $15^{\circ}$ diverging angle

duced by the DDT process may occupy only a very small percentage of one PDE cycle. Therefore, it can be negligible as long as it could complete inside the combustion chamber. However it may affect on PDE thermal efficiency.

\section{CONCLUSIONS}

Wave dynamics processes occurring in pulse detonation devices have been analyzed both numerically and experimentally, including the propagation of detonation, the motion of rarefaction waves in gas exhausting phase and the diffraction of shock wave at thrust nozzles. The observed wave phenomena are summarized as follows: the detonation precess occupies only a small percentage of one PDE cycle. The time taken due to the DDT precess may be negligible if detonative gas mixtures are pressurized at above $0.2 \mathrm{MPa}$. Rarefaction waves can exhaust detonation products to a very low pressure level but the temperature of remained exhaust gases is still quite higher. This is good for gas filling but auto-ignition of fresh gas mixture has to be considered. Numerical analysis shows that pulse detonation devices can be operated in a quite high frequency, the gas-filling phase occupies a longer time in one detonation cycle, and decreasing the length of detonation chamber for higher operation frequency is possible but its effect is limited.

\section{REFERENCES}

Chapman, D.L., 1899, "On the Rate of Explosions in 

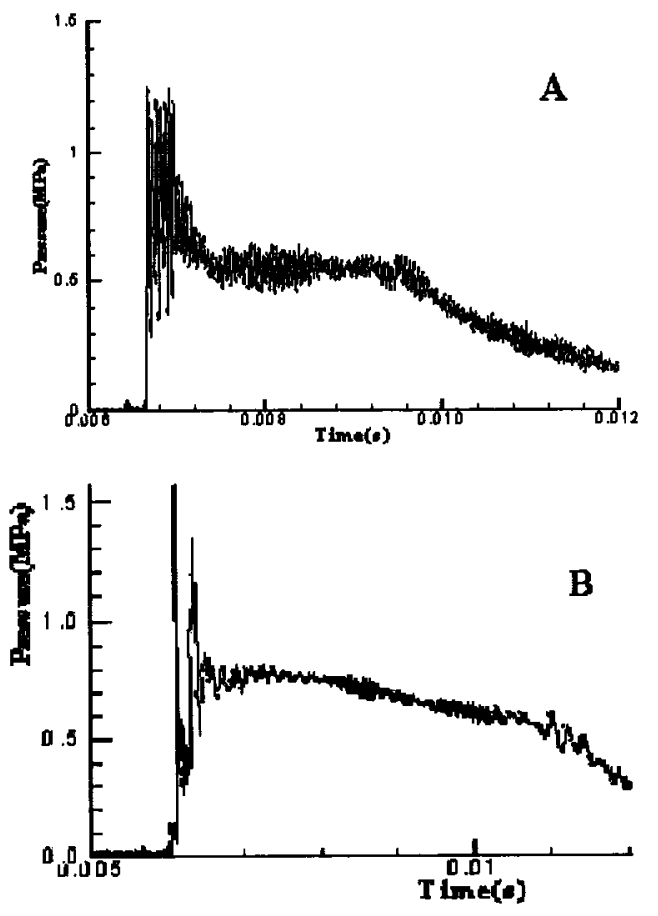

Figure 11: Pressure variations measured at position $A$ for two tests with the same initial conditions but different ignition energy

Gases", Phil. Mag., Vol. 47, p.90.

Jouguet, E., 1905, "Sur la Propagation des Reactions Chimiques Dans les Gaz", J. Maths. Pure Appl., Vol. 7, p.347.

Zel'dovich, Y. B., 1940, "On the Theory of the Propagation of Detonation in Gaseous Systems", J. Exp. Theor. Phys. USSR, Vol. 10, p. 524. Translation NACA Tech. Memo 1261.

von Neumann, J., 1942, "Progress Report on the Theory of Detonation Waves", OSRD Rept. No.549.
Doring, W., 1943, "Uber den Detonationsvorgang in Gasen", Ann. Physik, Vol.43, p.421.

Nettleton, M.A., 1987, "Gaseous Detonation, Their Natures, Effects and Control", Chapman and Hall, New York.

Hoffmann, H., 1940, "Reaction-Propulsion Produced by Intermittent Detonative Combustion, German Research Institute for Gliding", Rept.ATI-52365.

Nicholls, J. A., Wilkinson, H.R., and Morrison, R.B., 1957, "Intermittent Detonation as a Thrust-Producing Mechanism", Jet Propulsion, Vol. 27, No. 5, p. 534.

Krzychi, L.J., 1962, "Performance Characteristics on an Intermittent Detonative Combustion", AD-284312, U.S. Naval Ordnance Test Station, China Lake, CA.

Heldman, D., Shreeve, R. P., and Eildman, S., 1986, "Detonation Pulse Engines", AIAA Paper 86-1683.

Hinkey, J. B., Bussing, T.R.A., and Kaye, L., 1995, "Shock Tube Experiments for the Development of a Hydrogen-Fuelled Pulse Detonation Engines, AIAA Paper 95-2578.

Bussing, T.R.A., Hinkey, J.B., and Kaye, L., 1994, "Pulse Detonation Engine Preliminary Design Considerations", AIAA Paper 94-3220.

Kailasanath, K., 2000, "Review of Propulsion Applications of the Pulsed Detonation Waves", AIAA Journal, Vol. 38, No. 9, p. 1698-1708.

Brophy, C., Netzer, D. and Forster, D., 1998, "Detonation Studies of JP-10 with Oxygen and Air for Pulse Detonation Engine Development", AIAA paper 98-4003.

Jiang, Z.L., Falcovitz, J. and Takayama, K., 1997, "Numerical simulations of detonation in converging chambers", JSME International J, Vol. 40, pp. 422-431.

Jiang, Z.L., Takayama, K. and Chen, Y.S., 1995, "Dispersion conditions for non-oscillatory shock capturing schemes and its applications", Computational Fluid Dynamics J, Vol. 2, p. 137-150. 